\title{
New results on hard probes in heavy-ion collisions with LHCb
}

Frédéric Fleuret* on behalf of the LHCb collaboration.

LAL, Univ. Paris-Sud, CNRS/IN2P3, Université Paris-Saclay, Orsay, France

also at Laboratoire Leprince-Ringuet, CNRS/IN2P3, École polytechnique, Palaiseau, France

E-mail: fleuretein2p3.fr

Since 2013, the LHCb collaboration extended its physics programme to the study of ion collisions. Thanks to its unique tracking and PID capabilities in the forward rapidity region, the $\mathrm{LHCb}$ detector can provide important results on heavy flavour production in proton-lead and lead-lead collisions at LHC. Moreover, LHCb has the unique capability to operate in a fixedtarget configuration and study proton and ion-beam induced reactions on gaseous targets. In the following, we report on the results and performances on open and hidden heavy-flavour hadron production in proton-lead, lead-proton and lead-lead collisions, recorded in collider mode, and in proton-nucleus collisions, recorded in fixed target mode.

Sixth Annual Conference on Large Hadron Collider Physics (LHCP2018)

4-9 June 2018

Bologna, Italy

${ }^{*}$ Speaker. 


\section{Introduction}

Heavy quarks and quarkonia are recognized to be privileged probes of Quantum ChromoDynamics (QCD). Because of their mass - significantly larger than the $\Lambda_{Q C D}$ scale - they are excellent tools to investigate the rich interface between the perturbative and non-perturbative domain of QCD. Moreover, in the high temperature/density regime of QCD, they are decisive probes of the deconfined state of matter, the Quark Gluon Plasma (QGP) which can experimentally be studied in nucleus-nucleus collisions. Besides, the study of heavy flavour production in proton-nucleus collisions, where no QGP is formed, is needed for a correct interpretation of the results obtained in nucleus-nucleus collisions. This is crucial to establish a robust baseline in order to clearly identify phenomena specifically related to QGP. In addition, several cold nuclear matter effects can be studied in proton-nucleus collisions, such as: Parton shadowing or anti-shadowing of the nuclear parton distribution functions (nPDF) in the target nucleus [1], saturation effects [2], interaction with comoving hadrons [3], and parton energy loss [4, 5].

The LHCb detector [6] is a single-arm forward spectrometer covering the pseudorapidity range $2<\eta<5$, designed for the study of particles containing $b$ or $c$ quarks. The detector includes a high-precision tracking system consisting of a silicon-strip vertex detector (VELO) surrounding the interaction region, a large-area silicon-strip detector located upstream of a dipole magnet with a bending power of about $4 \mathrm{Tm}$, and three stations of silicon-strip detectors and straw drift tubes placed downstream of the magnet. Different types of charged hadrons are distinguished using information of two ring-imaging Cherenkov detectors and muons are identified by a system composed of alternating layers of iron and multiwire proportional chambers. The online event selection is performed by a trigger which consists of a hardware stage, based on information from the calorimeter and muon systems, followed by a two stages software trigger, which full event reconstruction in the second step.

\section{Heavy flavor production in $p \mathrm{~Pb}$ and $\mathrm{Pb} p$ collisions}
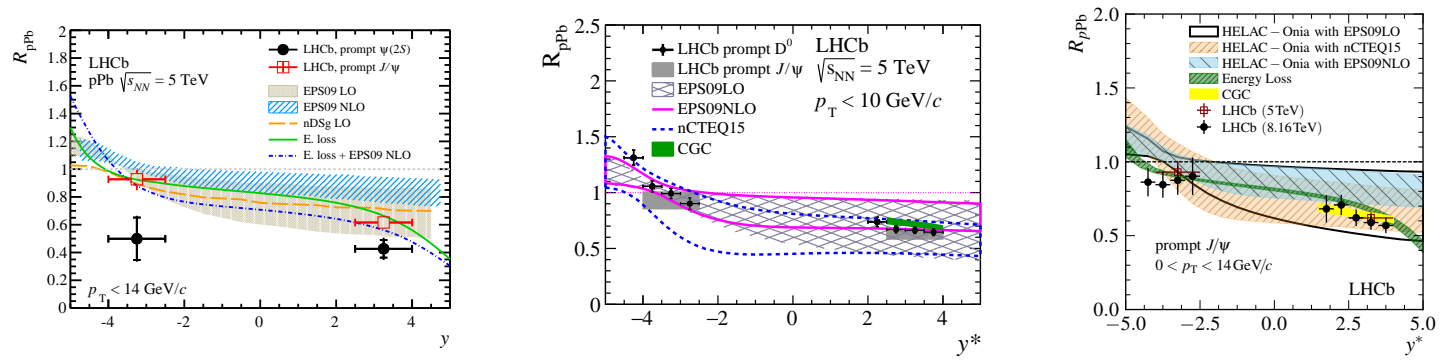

Figure 1: Nuclear modification factors $R_{p P b}$ in $p \mathrm{~Pb}$ and $\mathrm{Pb} p$ collisions as a function of the rapidity in the centre-of-mass system. Left: prompt J/ $\psi$ and prompt $\psi(2 S) R_{p P b}$ in $\sqrt{s_{N N}}=5 \mathrm{TeV}$ data. Mid: prompt $\mathrm{D}^{0}$ $R_{p P b}$ in $\sqrt{s_{N N}}=5 \mathrm{TeV}$ data. Right: prompt $\mathrm{J} / \psi R_{p P b}$ in $\sqrt{s_{N N}}=8 \mathrm{TeV}$ data.

In early 2013, the LHCb detector collected two data samples of $p \mathrm{~Pb}$ and $\mathrm{Pb} p{ }^{1}$ collisions at

\footnotetext{
${ }^{1}$ Either the proton beam or the lead beam is pointing along the beamline from the interaction point towards the $\mathrm{LHCb}$ detector, referred respectively as forward or backward configuration.
} 
$\sqrt{s_{N N}}=5 \mathrm{TeV}$, corresponding to an integrated luminosity of $1.6 \mathrm{nb}^{-1}$. Thanks to the inversion of the beams, LHCb covers both the forward $(y>0$ in $p \mathrm{~Pb}$ collisions) and backward $(y<0$ in $\mathrm{Pb} p$ collisions) rapidity regions. Fig. 1 (left plot) shows the nuclear modification factor $R_{p P b}$ of prompt $\mathrm{J} / \psi$ and prompt $\psi(2 S)$ as a function of the rapidity in the nucleon-nucleon centre-ofmass system [7, 8]. Prompt $\mathrm{J} / \psi$ results are in fair agreement with nPDF, coherent energy loss [9] and CGC [10] predictions. On the other hand, the $\psi(2 S)$ results show, in the backward region, a much larger suppression than expected by nPDF and coherent energy loss predictions. This additionnal suppression may be due to the interaction of the lightly-bound $\psi(2 S)$ state with the outcoming hadrons or partons produced in the collision [3]. Prompt $D^{0}$ mesons, measured in their hadronic decay channel, show a similar behavior as $\mathrm{J} / \psi$ and are in good agreement with nPDF and CGC predictions, as shown on Fig. 1 (middle plot). In 2016, two other samples of $p \mathrm{~Pb}$ and $\mathrm{Pb} p$ collisions have been recorded at $\sqrt{s_{N N}}=8 \mathrm{TeV}$ corresponding to an integrated luminosity of about $30 \mathrm{nb}^{-1}$. Thanks to this much larger samples, more precise results on prompt $\mathrm{J} / \psi$ production have been obtained [11]. These results confirm the pattern observed at lower energy and are in good agreement with nPDF, coherent energy loss and Color Glass Condensate (CGC) expectations, as shown on Fig. 1 (right plot). Thanks to the very good precision achieved, these results put strong constraints on the nPDF parametrizations [12].

Non-prompt $\mathrm{J} / \psi$, coming from the decay of $b$-hadrons, have been measured at both $\sqrt{s_{N N}}=5 \mathrm{TeV}$ and $\sqrt{s_{N N}}=8 \mathrm{TeV}$ (Fig. 2). They both show smaller nuclear matter effects than for prompt $\mathrm{J} / \psi$, in good agreement with EPS09NLO nPDF parametrizations [13]. The $R_{p P b}$ of $\Upsilon$, measured at $\sqrt{s_{N N}}$ $=5 \mathrm{TeV}$, shows a similar behaviour (Fig. 2 left).
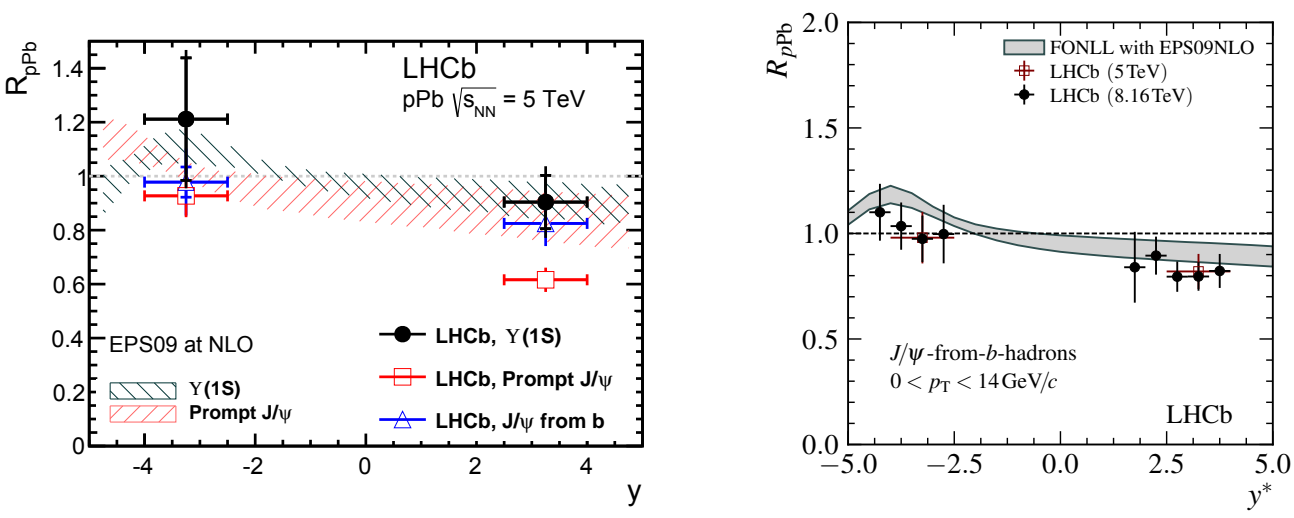

Figure 2: Nuclear modification factors $R_{p P b}$ as a function of $y^{*}$. Left: prompt $\mathrm{J} / \psi, \mathrm{J} / \psi$ from $b$-hadron decay and $\Upsilon R_{p P b}$ in $\sqrt{s_{N N}}=5 \mathrm{TeV}$ data. Right: $\mathrm{J} / \psi$ from $b$-hadron decay $R_{p P b}$ in $\sqrt{s_{N N}}=8 \mathrm{TeV}$ data.

The LHCb experiment has also measured prompt $\Lambda_{c}^{+}$production in $\sqrt{s_{N N}}=5 \mathrm{TeV} p \mathrm{~Pb}$ and $\mathrm{Pb} p$ collisions [14], extending the study of cold nuclear matter effects to charmed baryons. Fig. 3 shows the forward over backward ratio $R_{F B}$ defined as the ratio of the cross-sections in the forward and backward rapidity regions. The $R_{F B}$ as a function of both the transverse momentum $p_{T}$ (top left plot) and the rapidity in the centre-of-mass system $y^{*}$ are compatible with HELAC-Onia predictions $[15,16]$, which have been tuned to $p p$ data and include different nPDF sets. Charm baryon-tomeson ratio $R_{\Lambda_{c}^{+} / \mathrm{D}^{0}}$, the ratio of the $\Lambda_{c}^{+}$cross-section to the $\mathrm{D}^{0}$ cross-section, is also shown in Fig. 

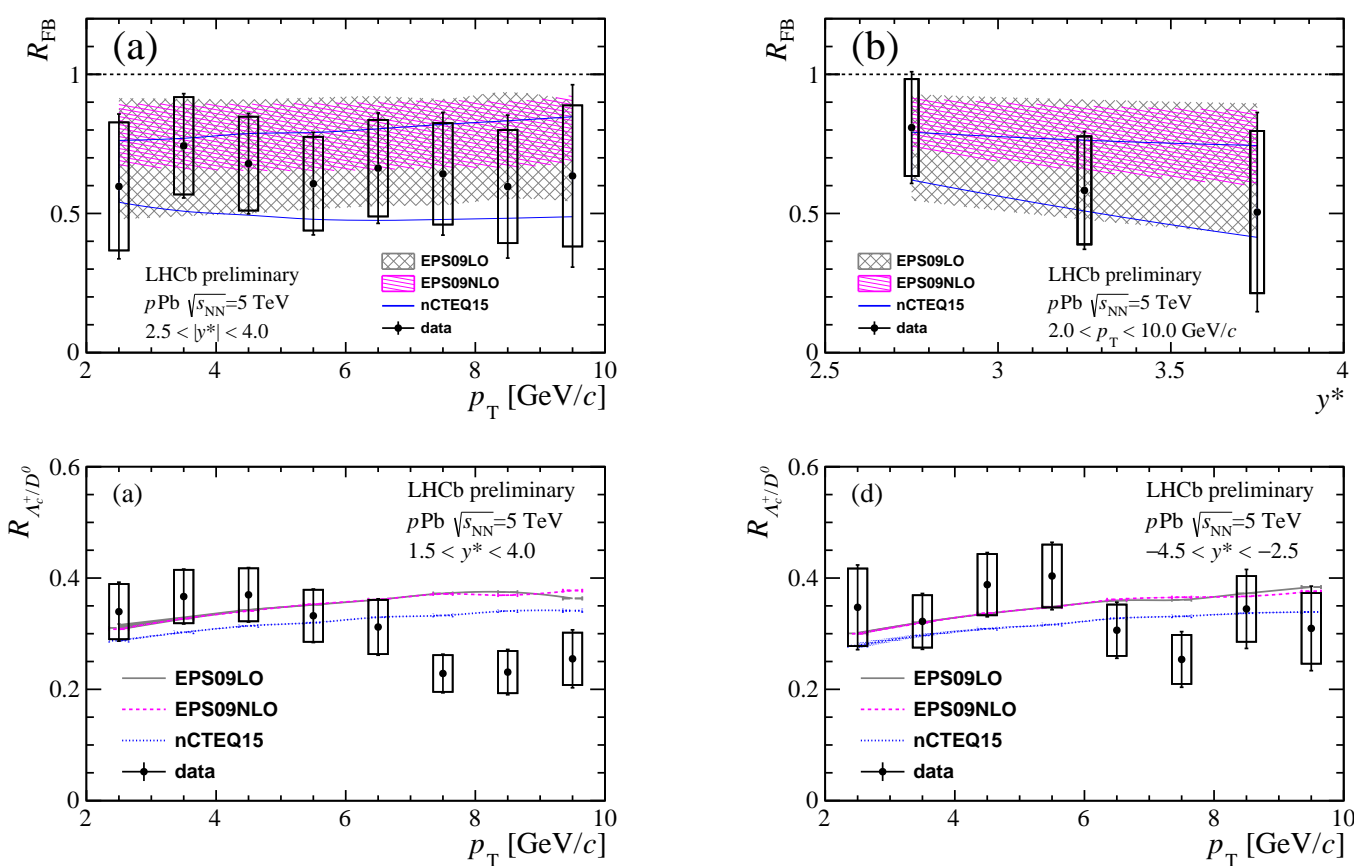

Figure 3: Top: Forward-to-backward ratio $R_{F B}$ of the $\Lambda_{c}^{+}$as a function of $p_{T}$ (left) and $y^{*}$ (right). Bottom: baryon-to-meson ratio $R_{\Lambda_{c}^{+} / \mathrm{D}^{0}}$ as a function of $p_{T}$ in the forward (left) and backward (right) rapidity region.

3. Since in such ratio, nPDF effects almost cancel, $R_{\Lambda_{c}^{+} / \mathrm{D}^{0}}$ is sensitive to the $c$ quark fragmentation. This ratio has been studied both in the forward (bottom left plot) and the backward (bottom right plot) rapidity region and compared to HELAC-Onia predictions which have been tuned to $p p$ data also for the fragmentation. Data are in general in good agreement with the calculations, except for the forward rapidity $p_{T}>7 \mathrm{GeV}$ region, where a small tension is observed.

\section{Heavy flavor production in PbPb collisions (Ultra-Peripheral Collisions)}

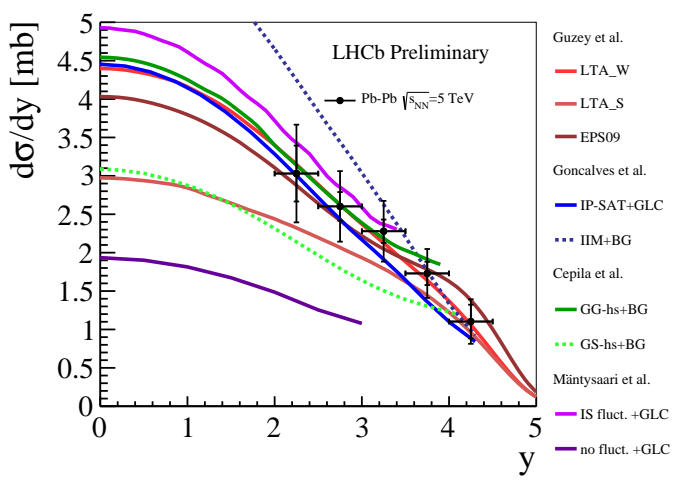

Figure 4: Coherent $\mathrm{J} / \psi$ production cross-section as a function of rapidity. Details on model calculation can be found in Ref. [17] 
$\mathrm{LHCb}$ participated for the first time in $\mathrm{PbPb}$ data taking in December 2015 collecting about 50 millions events at $\sqrt{s_{N N}}=5 \mathrm{TeV}$. In its current design, because of very high occupancy in most central $\mathrm{PbPb}$ events, the detector performances are limited to peripheral collisions. However, it is very well suited for the study of coherent $\mathrm{J} / \psi$ production in ultra-peripheral collisions where one ion interacts with the electromagnetic field of the other and where only two tracks reach the detector. This measurement is sensitive to the gluonic content of the nucleus. The coherent $\mathrm{J} / \Psi$ cross-section is found to be $\sigma_{\mathrm{J} / \psi}=5.27 \pm 0.21$ (stat) \pm 0.49 (syst) \pm 0.7 (lumi) $\mathrm{mb}$ [17], where the uncertainties are respectively statistical, systematic and related to the luminosity measurement. A comparison of the rapidity differential cross-section with theoretical predictions is shown in Fig. 4.

\section{Heavy flavor production at low energy (fixed-target mode)}
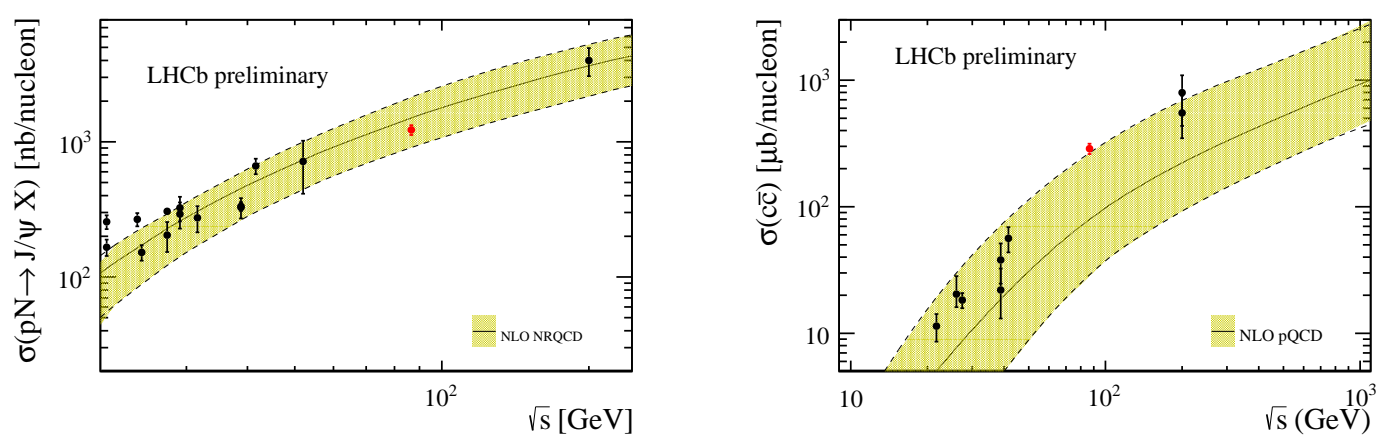

Figure 5: Left: $\mathrm{J} / \Psi$ cross-section measurements as a function of the centre-of-mass energy; Experimental data have been taken from Ref. [18]; The yellow band corresponds to a fit based on NLO NRQCD calculations. Right: $c \bar{c}$ cross-section measurements as a function of the centre-of-mass energy; Experimental data have been taken from Ref. [19]; The yellow band corresponds to NLO pQCD calculations. Red points correspond to the $\mathrm{LHCb} p \mathrm{He}$ results.

In LHCb, a System for Measuring Overlap with Gas (SMOG) is associated to the silicon-strip vertex locator (VELO) to measure the beam profile via its interaction with a very-low pressure injected gas. The SMOG allows the injection of noble gas such as $\mathrm{He}, \mathrm{Ne}, \mathrm{Ar}, \mathrm{Kr}$ and $\mathrm{Xe}$. It gives the unique opportunity to operate the LHCb detector in a fixed-target mode and to study nucleus-nucleus and proton-nucleus collisions on various targets at an nucleon-nucleon centre-ofmass energy close to $100 \mathrm{GeV}$. In such a regime, secondary charmonia production via statistical recombination doesn't occur, thus providing the possibility to directly test the thermal suppression of primary production.

First measurements of $\mathrm{J} / \psi$ and $\mathrm{D}^{0}$ production in this configuration has been performed with a $\sqrt{s_{N N}}=86.6 \mathrm{GeV}$ proton-helium sample recorded in 2016 and a $\sqrt{s_{N N}}=110.4 \mathrm{GeV}$ proton-argon sample recorded in 2015 [20]. The cross-section measurement has been performed for the $p \mathrm{He}$ sample only, since the luminosity determination could be performed so far only for this sample. The luminosity has been determined fron the yield of electrons elastically scattering off the target He atoms. Fig. 5 shows the $\mathrm{J} / \psi$ and $\mathrm{D}^{0}$ cross-sections measured in $p \mathrm{He}$ and their comparison with other measurements at various energies and with theoretical expectations.

$\mathrm{J} / \psi$ and $\mathrm{D}^{0}$ differential cross-sections in $p \mathrm{He}$ collisions, and yields in $p \mathrm{Ar}$ collisions, as functions 
of $y^{*}$ and $p_{T}$ have also been performed. Fig. 6 shows the $\mathrm{J} / \psi$ and $\mathrm{D}^{0}$ yields and cross-sections,
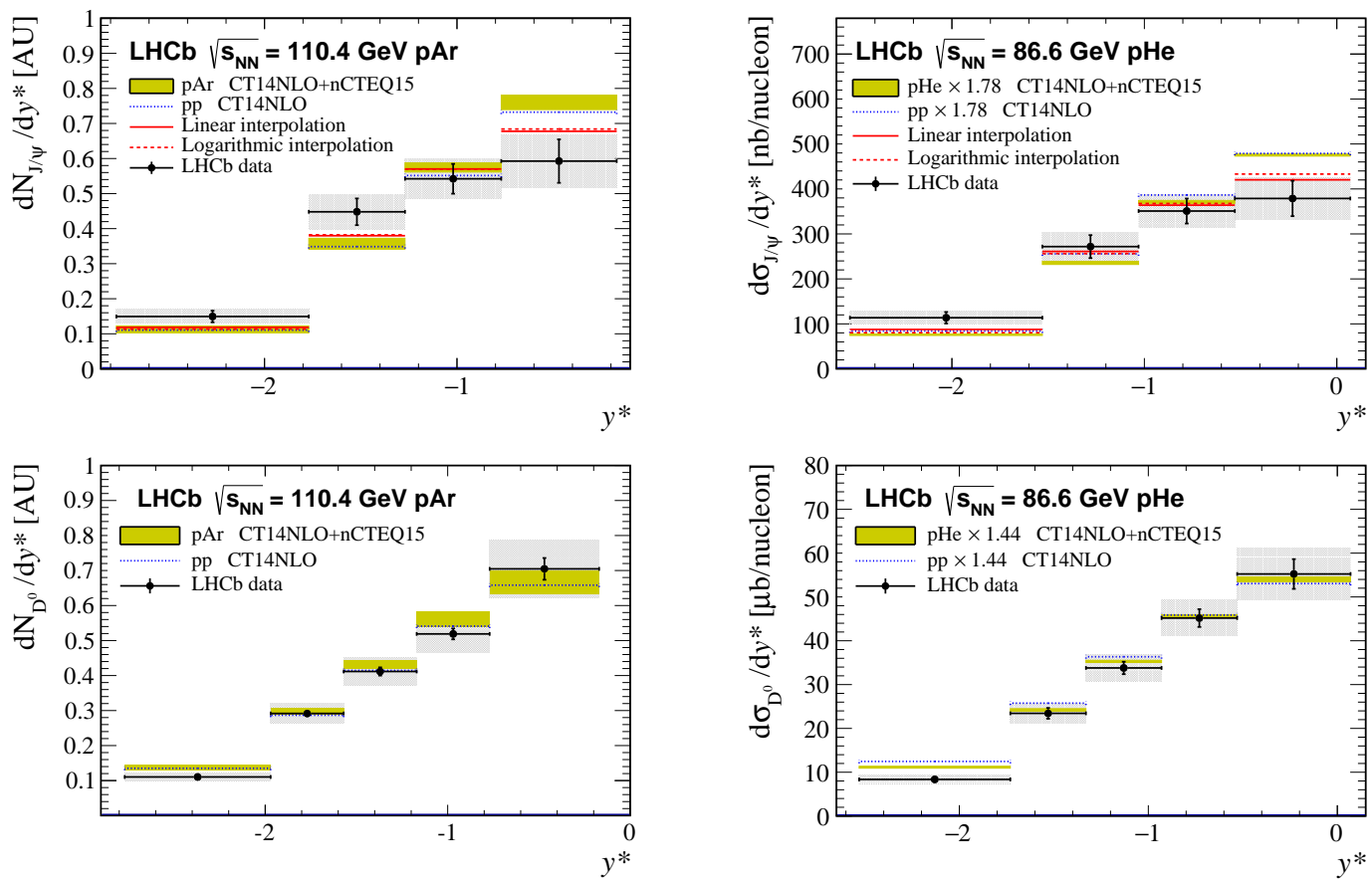

Figure 6: Differential $\mathrm{J} / \psi$ (top) and $\mathrm{D}^{0}$ (bottom) productions as a function of $y^{*}$ for the $p \mathrm{Ar}$ (left) and $p \mathrm{He}$ (right) data sets.

respectively for the $p \mathrm{Ar}$ and $p \mathrm{He}$ data. Data are compared with phenomenological and theoretical expectations. No strong differences in shapes are observed between $p \mathrm{He}$ data and their related theoretical predictions, showing no evidence of a strong valence-like intrinsic charm contribution in the most backward rapidity bin which covers the large Bjorken- $x$ region from $\sim 0.2$ to $\sim 0.4$.

\section{Conclusion}

The LHCb experiment has recently provided important results in heavy-ion collisions, giving, in particular, new constraints for a better understanding of heavy flavour production. Moreover, the LHCb experiment has the unique capability to perform a thorough heavy-ion program in a fixed target mode to disentangle charmonium suppression in heavy-ion collisions. Although the detector performances are currently limited to $\mathrm{PbPb}$ peripheral collisions, its performances should be improved after the upgrade of the tracking system which will occur during the next LHC Long Shutdown (LS2). Further improvements are envisioned for a longer term future.

\section{References}

[1] de Florian, D. and Sassot, R., Phys. Rev. D 69 (2004)

[2] J. L. Albacete and C. Marquet, Progress in Particle and Nuclear Physics 76 (2014) 1-42

[3] E. G. Ferreiro, Phys. Lett. B 749 (2015) 98

[4] Arleo, F. and Peigné, S., Phys. Rev. Lett. 109 (2012) 122301 
[5] Arleo, F. and Peigné, S. and Sami, T., Phys. Rev. D 83 (2011) 114036

[6] LHCb collaboration, A. A. Alves Jr. et al., JINST 3 (2008) S08005

[7] LHCb collaboration, R. Aaij et al., JHEP 02 (2014) 072

[8] LHCb collaboration, R. Aaij et al., JHEP 03 (2016) 133

[9] Arleo, F. and Peigné, S., JHEP 03 (2013) 122

[10] B. Ducloué, T. Lappi and H. Mantysaari, Phys. Rev. D 91, 114005

[11] LHCb collaboration, Phys. Lett. B 774 (2017) 159âĂŞ178

[12] Kusina, A. et al., Phys. Rev. Lett. 121 (2018) 052004

[13] K. J. Eskola, H. Paukkunen, C. A. Salgado, JHEP 04 (2009) 065

[14] LHCb collaboration, LHCb-CONF-2017-005, paper in preparation

[15] H.-S. Shao, Comput. Phys. Commun. 184 (2013) 2562

[16] H.-S. Shao, Comput. Phys. Commun. 198 (2016) 238

[17] LHCb collaboration, R. LHCb-CONF-2018-003.

[18] F. Maltoni et al., Phys. Lett. B638 (2006) 202

[19] ALICE collaboration, J. Adam et al., Phys. Rev. C94 (2016) 054908

[20] LHCb collaboration, LHCb-CONF-2017-001, paper in preparation 